\title{
Confinement-deconfinement transition in dense SU(2) QCD
}

\author{
V. G. Bornyakov ${ }^{1,2,3}$, V. V. Braguta ${ }^{1,2,4}$, E.-M. Ilgenfritz ${ }^{4}$, A. Yu. Kotov ${ }^{1,4,5}$, I. E. Kudrov ${ }^{1,5}$, \\ A. V. Molochkov ${ }^{2}$, A. A. Nikolaev ${ }^{1,2, \star}$, and R. N. Rogalyov ${ }^{3}$ \\ ${ }^{1}$ Institute for Theoretical and Experimental Physics NRC "Kurchatov Institute", Moscow, 117218 Russia \\ ${ }^{2}$ Far Eastern Federal University, School of Biomedicine, Vladivostok, 690950 Russia \\ ${ }^{3}$ Institute for High Energy Physics NRC "Kurchatov Institute", Protvino, 142281 Russia \\ ${ }^{4}$ Bogoliubov Laboratory of Theoretical Physics, Joint Institute for Nuclear Research, Dubna, 141980 Russia \\ ${ }^{5}$ Moscow Institute of Physics and Technology, Dolgoprudny, Moscow Region, 141700 Russia
}

\begin{abstract}
In this report we study the properties of the dense SU(2) QCD. The lattice simulations are carried out with improved gauge action and smaller lattice spacing as compared to our previous work. This allowed us to approach closer to the continuum limit and reach larger densities without lattice artifacts. We measured string tension and Polyakov loop as functions of chemical potential and temperature. At sufficiently large baryon density and zero temperature we observe confinement/deconfinement transition which manifests itself as a vanishing of the string tension and rising of the Polyakov loop.
\end{abstract}

\section{Introduction}

The knowledge of the properties of QCD at finite baryon density is very important for cosmology and astrophysics. A thorough experimental study of the quark-gluon matter is planned at future heavy ion collision experiments FAIR and NICA. Today, QCD at finite temperature and small baryon density is well explored thanks to lattice simulations. Unfortunately, lattice simulations can not be directly applied to the study of properties of the theory at sufficiently large baryon density because of the sign problem [1]. For this reason one has a rather poor knowledge about the QCD phase diagram in the region of large baryon density.

There are many phenomenological models which predict different interesting phenomena in this region of the phase diagram. As examples of such phenomena one should mention color flavor locking [2], non-uniform phases in dense matter [3], and more general features like the restoration of chiral symmetry [4] and deconfinement ${ }^{1}$ in dense QCD [6] etc. It is rather difficult to estimate systematic uncertainties of different phenomenological models. So, it is hard to assess if these phenomena are realized in the real world. On the other hand, in the absence of straightforward results from LQCD for the phase diagram one can study, for instance, QCD with isospin chemical potential [7-9].

In this paper we are going to study the deconfinement aspects of the transition(s) in dense quark matter at low temperature. Since in our consideration it is assumed that the temperature is much

\footnotetext{
^ Speaker, e-mail: nikolaev.aa@dvfu.ru

${ }^{1}$ Notice that the confinement/deconfinement and the chiral symmetry breaking/restoration transitions are not immediately related [5], and in dense matter they may take place at different densities.
} 
smaller than the baryon chemical potential, $T \ll \mu_{b}$, there is no hope that in the nearest future this region of the phase diagram will be reached by standard methods to overcome the sign problem in simulations of three-color QCD. Thus instead of considering the SU(3) gauge group, in this paper we are going to describe results of lattice simulation of two-color QCD. This theory is free from the sign problem and it can be directly studied on the lattice.

The SU(2) and SU(3) gauge theories have two important differences. The first one is that the chiral symmetry breaking pattern for the SU(3) gauge theory differs from that in the SU(2) theory [10]. The second difference is that baryons in the SU(3) theory contain three quarks and they are fermions whereas is the $\mathrm{SU}(2)$ theory baryons contain two quarks and they are bosons.

Despite these differences $S U(2)$ and $S U(3)$ gauge theories have a lot of common properties. In particular, in both theories confinement/deconfinement and chiral symmetry breaking/restoration transitions take place at a certain non-zero temperature. The mechanism of the fermion mass generation in a dense medium and even the formula for the fermion mass gap is the same in both theories [11]. In addition, many ratios of observables depend weakly on the number of colors [12].

Notice also that both in the region of sufficiently large chemical potential as well as at large temperature there is restoration of chiral symmetry. One can expect that in these regions the chiral symmetry breaking patterns (which differ in both theories) are not important. Moreover, in these regions of the phase diagram at large $\mu$ or $T$, where the relevant degrees of freedom are quarks and gluons it should be unimportant if baryons are composed of two or three quarks. Because of these arguments we believe that lattice simulation of the SU(2) QCD can be used not only for a qualitative study of dense matter but it can also give quantitative predictions for dense SU(3) QCD.

Many papers are devoted to lattice study of dense SU(2) QCD, see [13-17] and references therein. It is clear that in order to observe deconfinement in dense matter one needs to reach the region of sufficiently large quark chemical potential without being hampered by lattice artifacts. We believe that the largest value of the quark chemical potential $\mu_{q} \sim 800-1000 \mathrm{MeV}$ was safely reached in our previous paper $^{2}$ [15]. However, no signs of deconfinement were observed.

\section{Details of the simulation}

In this paper we continue our study of SU(2) QCD in the region of very large baryon density. In particular, we carry out lattice simulations with rooted staggered fermions which in the continuum limit corresponds to $N_{f}=2$ quark flavours. In order to observe condensation of scalar diquarks in a finite volume we introduced a diquark source term to the lattice action which is controlled by the parameter $\lambda$. In the paper [15] we observed that in the region of large baryon density our results weakly depend on the value of the parameter $\lambda$. In the present paper we have studied the dependence of our results on $\lambda$ only for few values of chemical potential $\mu_{q}$ and found that the sensitivity is indeed weak. So, to reduce the time of the simulations we restricted our consideration to the case $\lambda=0.00075$ which is much smaller than the bare quark mass $a m=0.0075$ used in the simulation.

Contrary to our previous study we used the Symanzik improved gauge action. In addition to the finite $\mu_{q}$ simulations also calibration simulations at zero chemical potential have been performed. We used the QCD Sommer scale $r_{0}=0.468(4)$ fm [18] to carry out the scale setting. In this case our string tension at $\mu_{q}=0$ is $\sqrt{\sigma_{0}}=476(5) \mathrm{MeV}$ at $a=0.044 \mathrm{fm}$ whereas in our previous paper [15] the lattice spacing was almost three times larger, $a=0.112 \mathrm{fm}$. This change allowed us to approach closer the continuum limit and reach larger baryon densities without being hampered by lattice artifacts. In particular, in the present paper we reach the region of baryon density with a quark chemical potential $\mu_{q}>2000 \mathrm{MeV}$ which is the largest value ever reached in lattice simulation of SU(2) QCD.

\footnotetext{
${ }^{2}$ Note that throughout this paper we express our results as functions of the quark chemical potential $\mu_{q}$. As we study the theory with the gauge group $\mathrm{SU}(2)$, this corresponds to baryon chemical potential $\mu_{b}=2 \mu_{q}$.
} 
The simulations are performed on a $32^{4}$ lattice (compare with the lattice $16^{3} \times 32$ in our previous study [15]) and on $32^{3} \times 24,32^{3} \times 16,32^{3} \times 8$ lattices to study the finite temperature effects. Numerical simulation in the region of large baryon density requires considerable computer resources. For this reason, for the present paper we conducted our study at a pion mass of $m_{\pi}=740(40) \mathrm{MeV}$, a value which is larger than that used in [15]. We are going to decrease the pion mass in our future simulations. In our present study we have $m_{\pi} L_{s} \simeq 5$ to be compared with $m_{\pi} L_{s} \simeq 3$ in our previous study. So, the pion mass is larger but finite volume effects are better under control in the present study.

\section{Numerical results and discussion}

We start the presentation of the results of our study with the measurements of the average Polyakov loop $\langle L\rangle$. To measure it one step of HYP smearing [19] was employed for temporal links with the smearing parameters of the HYP2 parameter set [20]. Without the HYP smearing the Polyakov loop is zero within the uncertainty of the calculation.

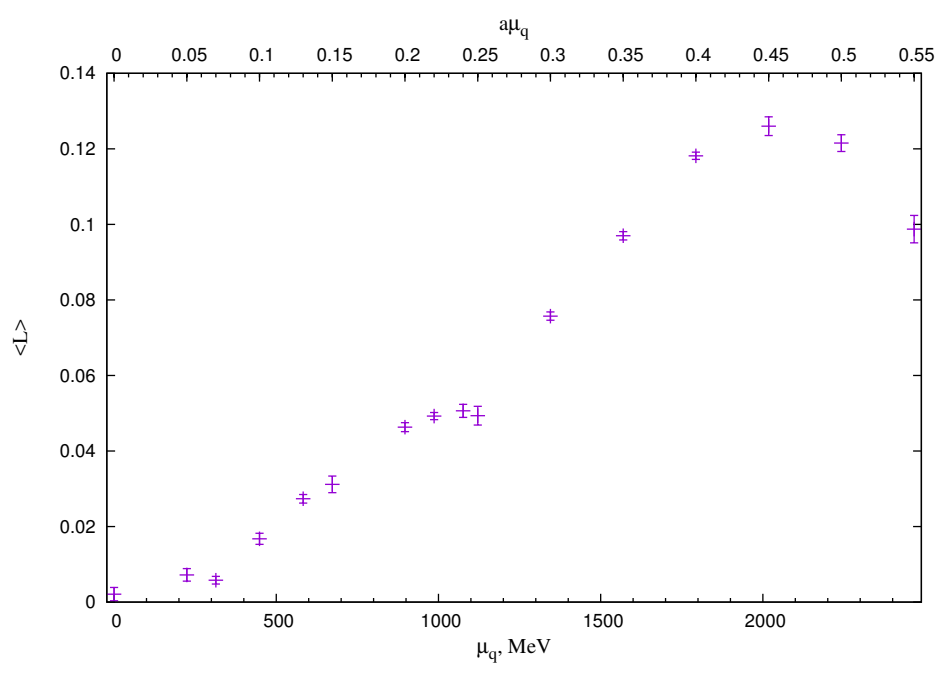

Figure 1. The Polyakov loop $\langle L\rangle$ as a function of the quark chemical potential. The quark chemical potential is shown in physical and lattice units.

The result of the measurement of the Polyakov loop is shown in Fig. 1. It is seen from this plot that - contrary to the behaviour of the Polyakov loop at the temperature-driven confinement/deconfinement transition where it is a monotonous function of temperature - the dependence of the Polyakov loop on the chemical potential is a rather complicated function. First it rises up to $\mu_{q} \simeq 900 \mathrm{MeV}\left(a \mu_{q}=0.2\right)$, then it goes over to some plateau. After the plateau at $\mu_{q} \simeq 1100 \mathrm{MeV}\left(a \mu_{q}>0.25\right)$ the Polyakov loop rises again reaching maximum at $\mu_{q} \simeq 2000 \mathrm{MeV}\left(a \mu_{q}=0.45\right)$ and then it drops. It is not quite clear what physical phenomena are hidden behind this highly nontrivial behaviour of the Polyakov loop.

To study a possible deconfinement transition in dense matter we measured the interaction potential between a static quark-antiquark pair through the measurement of the Wilson loops. To measure it one step of HYP smearing [19] was employed for temporal links with the smearing parameters of the HYP2 parameter set [20], followed by 24 steps of APE smearing [21] for spatial links only with the 


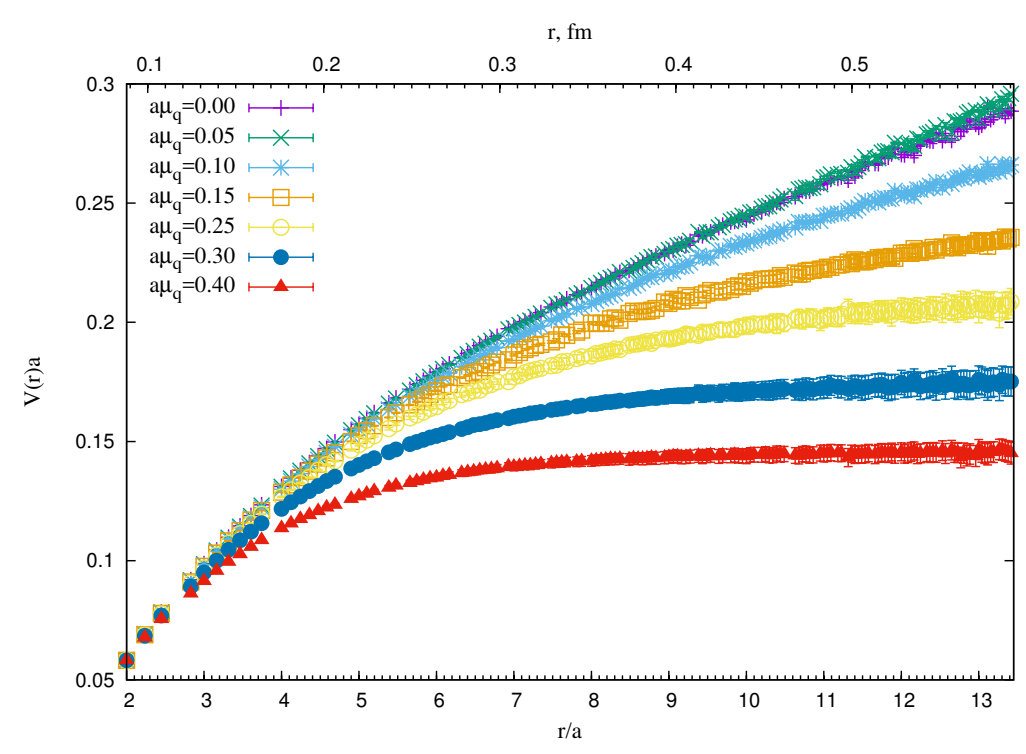

Figure 2. The interaction potential between static quark-antiquark pair $V(r)$ in lattice units through the measurement of Wilson loops. The distance between the static quark-antiquark pair is measured in lattice units.

smearing parameter $\alpha_{A P E}=0.25$. The result of this measurement is shown in Fig. 2. From this figure it is seen that for sufficiently small $\mu_{q}$ the potential $V(r)$ is a linearly rising function of distance, i.e. the system is in the confinement phase. For large values of $\mu_{q}$ the potential $V(r)$ at large distance goes to plateau, i.e. the system is in the deconfinement phase. Thus we observe a confinement/deconfinement transition in dense matter.

Further let us determine the chemical potential characterizing the transition from the confinement to the deconfinement phase. To do this we find the string tension $\sigma$ as a function of the chemical potential through a fit of our data to the Cornell potential $V(r)=\alpha / r+\sigma r+c$. In the fitting procedure we impose the condition $\sigma \geq 0$. It turns out that at nonzero chemical potential the value of $\sigma$ strongly depends on the region (in distance $r$ ) where we fit our data. So, in addition to the statistical uncertainty there is an uncertainty due to the variation of the fitting region. To estimate this source of uncertainty we varied the fitting region from $r / a \in[4,15]$ to $r / a \in[6,15]$, considered the string tension for the region $r / a \in[5,15]$ as the final value and added variation related to the change of the fitting range into the error. The fit is good for not very large values of the chemical potential. For a chemical potential $\mu_{q} \geq 1300 \mathrm{MeV}$ the Cornell potential does not describe our data at all.

In Fig. 3 we plot the string tension as a function of the chemical potential. From Fig. 3 it may be seen that the string tension $\sigma$ decreases with increasing chemical potential. Thus we see that the system becomes less confined in dense matter. Finally in the region $\mu_{q} \geq 900 \mathrm{MeV}\left(a \mu_{q} \geq 0.2\right)$ within the uncertainty of the calculation the string tension is zero. So, we conclude that the deconfinement takes place in the region $\mu_{q} \geq 900 \mathrm{MeV}$.

As was mentioned above the Cornell potential describes our data quite well in the region of sufficiently small chemical potential and it does not describe the data at large chemical potential. We believe that this happens since at large chemical potential the system under study is in the deconfinement phase where the Cornell potential is not applicable. It is known from $\mu_{q}=0$ studies that in the deconfinement phase the static potential of a quark-antiquark pair can be desribed by the Debye 


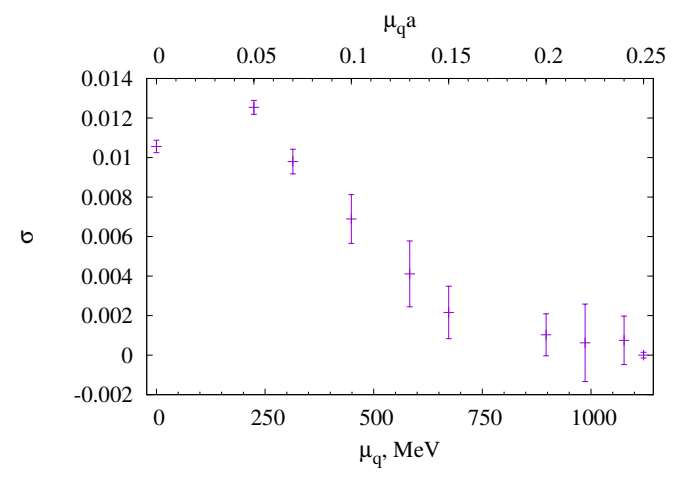

Figure 3. String tension $\sigma$ as a function of the quark chemical potential. The quark chemical potential $\mu_{q}$ is shown in physical and lattice units.

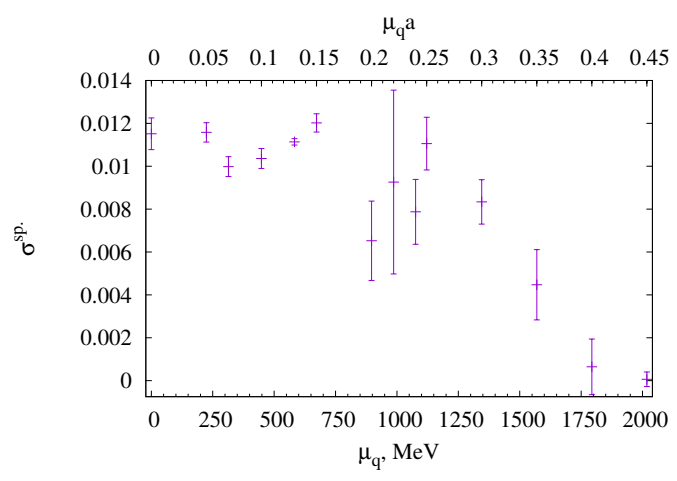

Figure 4. Spatial string tension $\sigma_{s}$ as a function of the chemical potential. The quark chemical potential $\mu_{q}$ is shown in physical and lattice units.

screened potential $V(r)=(a / r) e^{-m_{D} r}+c$. We tried to describe our data by a Debye screened Coulomb potential in the region $r / a \in[4,15]$ and found that the fit is good for the values $\mu_{q} \geq 1300 \mathrm{MeV}$ $\left(a \mu_{q} \geq 0.3\right)$. In the region $\mu_{q}<1300 \mathrm{MeV}$ the Debye potential does not describe our data.

From this consideration one can conclude that the confinement/deconfinement transition takes place in the region $\mu_{q} \in(900,1300) \mathrm{MeV}$. Below we will take the midpoint of this interval $\mu_{q} \sim$ $1100 \mathrm{MeV}$ as an estimate of the position of the transition.

A more accurate determination of the confinement/deconfinement transition in dense matter can be done through the measurement of the susceptibility of the Polyakov loop as well as with other susceptibilities. Unfortunately we are not able to do this since this would require huge statistics what is not accessible within our present resources.

To get more insight about the confinement/deconfinement transition in dense matter let us study the dependence of the spatial string tension $\sigma_{s}$ on the chemical potential. To do this we measured spatially oriented Wilson loops. Taking one spatial direction as a "time" direction, one can use the relation between the Wilson loop and the static potential and determine a "spatial potential" $V_{s}(r)$. To determine the spatial string tension we fit this function $V_{s}(r)$ by the Cornell parametrization $V_{s}(r)=$ $\alpha_{s} / r+\sigma_{s} r+c_{s}$. For all values of the chemical potential under study this form of Cornell potential fits our data well. The fitting parameter $\sigma_{s}$ is the spatial string tension. Indeed at large distance the spacelike potential $V_{s}(r)$ rises as $\sigma_{s} r$, what implies that the spatial Wilson loop $W_{s}$ behaves as $W_{s} \sim \exp \left(-\sigma_{s} A\right)$, where $A$ is a area of the surface spanned by the Wilson loop. Thus we determine the spatial string tension.

In Fig. 4 we plot the spatial string tension as a function of the chemical potential. From this Figure one can see a monotonous decrease of the spatial string tension in the region $\mu_{q} \geq 1100 \mathrm{MeV}\left(a \mu_{q} \geq\right.$ $0.25)$. Notice that this decrease starts precisely in the region where we have observed the conventional (timelike) confinement/deconfinement transition in our system. The monotonous decrease ends at $\mu_{q} \sim 2000 \mathrm{MeV}\left(a \mu_{q} \sim 0.45\right)$ where the spatial string tension has become zero within the uncertainty of the calculation. Thus starting from $\mu_{q} \sim 2000 \mathrm{MeV}$ spatial confinement disappears.

To understand the physical meaning of this result let us recall that the confinement/deconfinement transition at finite temperature and zero chemical potential is connected with the disappearence of the timelike string tension. On the opposite, after the transition the spatial string tension does not disap- 
pear. Contrary, it rises with the temperature, and the spatial potential in QCD is "would-be confining" at any temperature [22]. This means that there are nonperturbative effects (in the magnetic sector) in zero density QCD for any temperature. From this perspective, the confinement/deconfinement transition at finite density and zero temperature looks quite different. Similarly we observe vanishing of the "timelike" string tension. After this the spatial string tension starts to decrease and at the chemical potential $\mu_{q} \sim 2000 \mathrm{MeV}$ it also vanishes. Notice that the vanishing of both string tensions might indicate the disappearance of all nonperturbative effects in QCD. At the same time, due to asymptotic freedom, the coupling constant is already sufficiently small in the region $\mu_{q} \geq 2000 \mathrm{MeV}$. So, one can conjecture that in the region beyond $\mu_{q} \sim 2000 \mathrm{MeV}$ the quark-gluon plasma is similar to a weakly interacting gas of quarks and gluons.

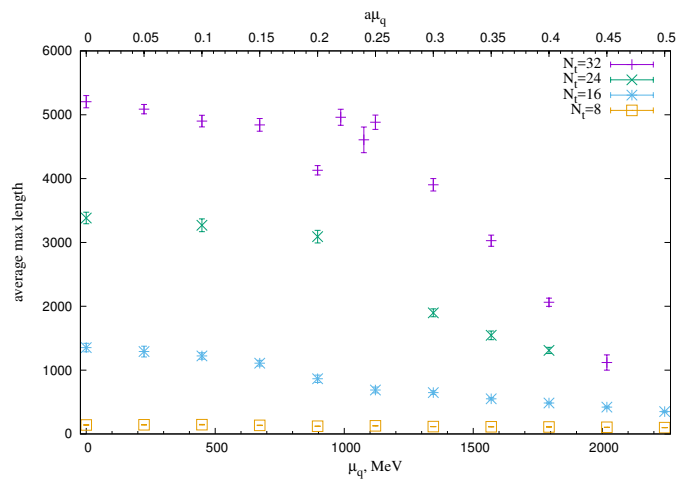

Figure 5. Average length of maximal cluster for different $N_{t}$. The quark chemical potential $\mu_{q}$ is shown in physical and lattice units.

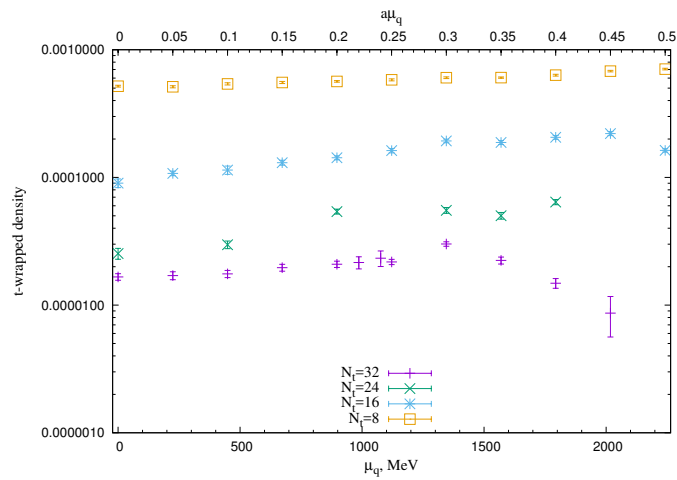

Figure 6. The density of monopoles trajectories (logarithmic scale) wrapped around the temporal extent of the lattice for different $N_{t}$. The quark chemical potential $\mu_{q}$ is shown in physical and lattice units.

The picture of confinement/deconfinement transition in dense matter observed so far in this paper is also supported by our study of Abelian monopoles which are revealed by Abelian projection. It is known that the properties of the Abelian monopoles are related to the confinement/deconfinement transition. In particular, in the confinement phase there is a percolating cluster of monopole currents which disappears at the transition to deconfinement [23]. The same behaviour takes place in dense matter. In confinement we observe always a percolating cluster which disappears in the region $\mu_{q} \geq$ $1100 \mathrm{MeV}$, see Fig. 5. For $N_{t}=32(T=0)$ the disappearance of the percolating cluster is noticeable, but for finite temperatures it seems to be smooth and for the highest temperature there is no percolating cluster at all. It should be added that at high temperature the spatial string tension is related to the density of monopoles trajectories wrapped around the (periodic) temporal extent of the lattice. The larger the density of wrapped monopoles trajectories the larger the spatial string tension. In our study of dense SU(2) QCD we observe that after the standard timelike confinement/deconfinement transition at $\mu_{q} \geq 1100 \mathrm{MeV}$ the density of wrapped monopoles trajectories starts to decrease (see Fig. 6 for $N_{t}=32$ ). This behaviour is in agreement with the decrease of the spatial string tension in the same region. One can also observe that finite temperature destroys this effect.

Our study of the confinement/deconfinement transition was performed within lattice simulation of SU(2) QCD. At the end of this paper let us discuss the validity of our results for the case of SU(3) QCD. As was noted above there are two very important differences between two-color and three- 
color QCD. The first one is that the chiral symmetry breaking pattern of both theories is different. The second one is that baryons in the SU(3) theory are fermions and contain three quarks whereas is the $\mathrm{SU}(2)$ theory baryons are bosons and contain two quarks. According to our results the confinement/deconfinement transition takes place at very large baryon density $\left(\mu_{q} \sim 1100 \mathrm{MeV}\right)$. Notice that in this region of $\mu_{q}$ the chiral symmetry is already restored [15]. So, we believe that the chiral symmetry breaking pattern does not play any important role in this region. Moreover, according to our previous results [15] in the region of large baryon density the key degrees of freedom are quarks rather than baryons. These facts allow us to conjecture that the confinement/deconfinement transition in the SU(3) theory at $T=0$ takes place in the region $\mu_{q} \sim 1000 \mathrm{MeV}$. We can also expect that the physical scenario of the transition in the SU(3) theory is similar to that in the SU(2) case. In particular, we expect that the vanishing of the timelike string tensition is followed by the vanishing of the spatial string tension at sufficiently larger baryon density also in SU(3) QCD.

\section{Conclusions}

In conclusion, in this paper we have studied the confinement/deconfinement transition aspects in dense SU(2) QCD within lattice simulation. The simulations were performed on a space-time symmetric lattice $32^{4}$ with rooted staggered fermions at lattice spacing $a=0.044 \mathrm{fm}$. The small lattice spacing has allowed us to reach the region of very large baryon density $\left(\mu_{q}>2000 \mathrm{MeV}\right)$ avoiding lattice artifacts. We have measured the Polyakov loop, the interaction potential between static quark-antiquark pair, the string tension and the spatial string tension for different values of the baryonic chemical potential.

In the region $\mu_{q} \sim 1100 \mathrm{MeV}$ we have observed the confinement/deconfinement transition which manifests itself in rising of the Polyakov loop and vanishing of the string tension. After the onset of deconfinement $\mu_{q}>1100 \mathrm{MeV}$ we have observed a monotonous decrease of the spatial string tension which ends up with the vanishing of the spatial string tension in the region $\mu_{q}>2000 \mathrm{MeV}$. So, in total, the confinement/deconfinement transition at finite density and zero temperature is quite different from that at finite temperature and zero density. In addition one can expect that in the region of very dense matter the quark-gluon plasma is similar to a weakly interacting gas of quarks and gluons, what differs from the quark-gluon plasma at large temperature.

\section{Acknowledgments}

The work was supported by RFBR grants 16-02-01146 A and 16-32-00048. This work has been carried out using computing resources of the federal collective usage center Complex for Simulation and Data Processing for Mega-science Facilities at NRC "Kurchatov Institute", http://ckp.nrcki.ru/. In addition we used the supercomputer of Institute for Theoretical and Experimental Physics (ITEP). 


\section{References}

[1] S. Muroya, A. Nakamura, C. Nonaka, T. Takaishi, Prog. Theor. Phys. 110, 615 (2003), hep-lat/0306031

[2] M.G. Alford, K. Rajagopal, F. Wilczek, Nucl. Phys. B537, 443 (1999), hep-ph/9804403

[3] T. Kojo, Y. Hidaka, L. McLerran, R.D. Pisarski, Nucl. Phys. A843, 37 (2010), 0912 . 3800

[4] M.G. Alford, K. Rajagopal, F. Wilczek, Phys. Lett. B422, 247 (1998), hep-ph/9711395

[5] H. Suganuma, T.M. Doi, K. Redlich, C. Sasaki (2017), 1709.05981

[6] L. McLerran, R.D. Pisarski, Nucl. Phys. A796, 83 (2007), 0706. 2191

[7] P. de Forcrand, M.A. Stephanov, U. Wenger, PoS LAT2007, 237 (2007), 0711.0023

[8] G. Endrödi, Phys. Rev. D90, 094501 (2014), 1407.1216

[9] P. Scior, L. von Smekal, D. Smith, Spectrum of QCD at Finite Isospin Density, in Proceedings, 35th International Symposium on Lattice Field Theory (Lattice2017): Granada, Spain, to appear in EPJ Web Conf., 1710.06314

[10] J.B. Kogut, M.A. Stephanov, D. Toublan, J.J.M. Verbaarschot, A. Zhitnitsky, Nucl. Phys. B582, 477 (2000), hep-ph/0001171

[11] D.T. Son, Phys. Rev. D59, 094019 (1999), hep-ph/9812287

[12] B. Lucini, M. Panero, Phys. Rept. 526, 93 (2013), 1210. 4997

[13] J.B. Kogut, D. Toublan, D.K. Sinclair, Nucl. Phys. B642, 181 (2002), hep-lat/0205019

[14] S. Cotter, P. Giudice, S. Hands, J.I. Skullerud, Phys. Rev. D87, 034507 (2013), 1210.4496

[15] V.V. Braguta, E.M. Ilgenfritz, A.Yu. Kotov, A.V. Molochkov, A.A. Nikolaev, Phys. Rev. D94, 114510 (2016), 1605.04090

[16] L. Holicki, J. Wilhelm, D. Smith, B. Wellegehausen, L. von Smekal, PoS LATTICE2016, 052 (2017), 1701.04664

[17] O. Hajizadeh, T. Boz, A. Maas, J.I. Skullerud, Gluon and ghost correlation functions of 2-color QCD at finite density, in Proceedings, 35th International Symposium on Lattice Field Theory (Lattice2017): Granada, Spain, to appear in EPJ Web Conf., 1710.06013

[18] A. Bazavov et al., Phys. Rev. D85, 054503 (2012), 1111.1710

[19] A. Hasenfratz, F. Knechtli, Phys. Rev. D64, 034504 (2001), hep-lat/0103029

[20] M. Della Morte, A. Shindler, R. Sommer, JHEP 08, 051 (2005), hep-lat/0506008

[21] M. Albanese et al. (APE), Phys. Lett. B192, 163 (1987)

[22] M. Cheng et al., Phys. Rev. D78, 034506 (2008), 0806. 3264

[23] V.G. Bornyakov, V.K. Mitrjushkin, M. Muller-Preussker, Phys. Lett. B284, 99 (1992) 